\title{
Discrete decay of the yrast superdeformed band in the ${ }^{151} \mathrm{~Tb}$ nucleus
}

J. Robin,,${ }^{1,2,}$ A. Odahara,,${ }^{1,3}$ G. Duchêne, ${ }^{1}$ F. A. Beck,${ }^{1}$ P. Twin,${ }^{4}$ Th. Byrski, ${ }^{1}$ D. Curien,,${ }^{1}$ N. Adimi,${ }^{5}$ D. E. Appelbe,${ }^{6}$ P. Bednarczyk, ${ }^{1,7}$ A. Bracco ${ }^{8}$ B. Cederwall, ${ }^{9}$ S. Courtin, ${ }^{1}$ D. M. Cullen,${ }^{4}$ O. Dorvaux,${ }^{1}$ S. Ertück, ${ }^{4,10}$ G. de France, ${ }^{1,11}$ B. Gall, ${ }^{1}$ P. Joshi, ${ }^{1,12}$ S. L. King, ${ }^{4}$ A. Korichi, ${ }^{13}$ K. Lagergren, ${ }^{9}$ G. Lo Bianco, ${ }^{14}$ S. Leoni, ${ }^{8}$ A. Lopez-Martens, ${ }^{13}$ S. Lunardi,${ }^{15}$ B. Million, ${ }^{8}$ A. Nourredine, ${ }^{1}$ E. Pachoud, ${ }^{1}$ E. S. Paul,${ }^{4}$ C. Petrache,,${ }^{14,16}$ I. Piqueras,${ }^{1}$ N. Redon, ${ }^{17}$ A. Saltarelli, ${ }^{14}$ J. Simpson, ${ }^{6}$ O. Stezowski, ${ }^{17}$ R. Venturelli, ${ }^{15}$ J. P. Vivien,,${ }^{1,}$ and K. Zuber $^{7}$

${ }_{1}^{1}$ Institut de Recherches Subatomiques, UMR 7500, IN2P3-CNRS-ULP, F-67037 Strasbourg Cedex 2, France

${ }^{2}$ LPSC, Université Joseph Fourier Grenoble 1, CNRS/IN2P3, Institut National Polytechnique de Grenoble, F-38026 Grenoble Cedex, France ${ }^{3}$ Department of Physics, Osaka University, Toyonaka, Osaka 560-0043, Japan

${ }^{4}$ Oliver Lodge Laboratory, University of Liverpool, Liverpool L69 7ZE, United Kingdom

${ }^{5}$ USTHB, Institut de Physique, BP32, El Alia, Bab Ezzouar, 16111 Alger, Algeria

${ }^{6} C C L R C$, Daresbury Laboratory, Daresbury Warrington WA4 4AD, United Kingdom

${ }^{7}$ Institut of Nuclear Physics, PL-31-42 Kraków, Poland

${ }^{8}$ Dipartimento di Fisica dell' Università di Milano and INFN, Sezione di Milano, I-20133 Milano, Italy

${ }^{9}$ Royal Institute of Technology, Alba Nova University Center, S-106 91 Stockholm, Sweden

${ }^{10}$ Nigde University, Science and Art Faculty, Department of Physics, 51100 Nigde, Turkey

${ }^{11}$ Grand Accélérateur National d'Ions Lourds (GANIL), CEA/DSM-CNRS/IN2P3, Bd Henri Becquerel, BP 55027, F-14076 Caen Cedex 5, France

${ }^{12}$ Department of Physics, University of York, Heslington, York YO10 5DD, United Kingdom

${ }^{13}$ C. S. N. S. M., IN2P3-C. N. R. S, F-91405 Orsay Campus, France

${ }^{14}$ Dipartimento di Fisica dell' Università di Camerino and INFN, Sezione di Camerino, I-62032 Camerino, Italy

${ }^{15}$ Dipartimento di Fisica dell' Università di Padova and INFN, Sezione di Padova, I-35131 Padova, Italy

${ }^{16} I P N$, IN2P3-CNRS and Université Paris-Sud, F-91406 Orsay, France

${ }^{17}$ Institut de Physique Nucléaire, F-69622 Villeurbanne Cedex, France

(Received 4 April 2008; published 29 September 2008)

The Euroball array has been used to search for linking transitions between the superdeformed (SD) and the normal deformed (ND) wells in ${ }^{151} \mathrm{~Tb}$. Many $\gamma$ rays in the energy range $2-4 \mathrm{MeV}$ have been observed in coincidence with the yrast SD band. It is proposed that the highest energy transition of $3748 \mathrm{keV}$ and the strongest line $(2818 \mathrm{keV})$ of the previously observed transitions both decay from the same SD level as their links with the ND states have been identified. The current spectra have insufficient statistics to completely identify the decay point in the SD band. Theoretical calculations covering SD bands in the $A \approx 150$ region enable the two possible spin assignments to be compared with experimental data on proposed links in ${ }^{149} \mathrm{Gd}$ and ${ }^{152} \mathrm{Dy}$. It is concluded that the energy of the lowest SD band member in ${ }^{151} \mathrm{~Tb}$ has an excitation energy of $12861 \mathrm{keV}$ and a spin of $65 / 2^{+}$.

DOI: 10.1103/PhysRevC.78.034319

PACS number(s): 21.10.Re, 23.20.En, 23.20.Lv, 27.70.+q

\section{INTRODUCTION}

Since the first observation in the ${ }^{152}$ Dy nucleus in 1986 [1] more than $\sim 250$ superdeformed (SD) bands have been identified in various mass regions extending from $A \approx 30$ to $A \approx 190$ [2]. Many of the new SD bands found in the $A \approx 40,60,80$, and 130 mass regions were easily connected to normally deformed states because of a smaller change in deformation between the SD and ND states and due to a lower level density in the ND well. However, in the $A \approx 150$ and $A \approx 190$ mass regions the decay is dominated by its statistical aspects. This results in the decay-out contribution to the spectrum being dominated by a quasicontinuum with very weak discrete linking transitions between SD and ND wells. So, despite the large number of SD bands discovered in the

\footnotetext{
*robin@1psc.in2p3.fr

†Deceased.
}

$A \approx 150$ and $A \approx 190$ mass regions only few linking transitions connecting SD bands to normally deformed (ND) states have been found. First evidence of such linking transitions were reported in ${ }^{194} \mathrm{Hg}$ [3] and ${ }^{192,193,194} \mathrm{~Pb}$ isotopes [4-6]. In the $A \approx 150$ region there have only been two reports of links; a weak two-step decay in ${ }^{149} \mathrm{Gd}$ [7] and a single-step transition in ${ }^{152}$ Dy [8]. There have been many theoretical calculations of SD bands in the $A \approx 150$ region and, in particular, calculations [9] of the relative alignments enable a comparison of spins in different nuclei. These indicate that the ${ }^{149} \mathrm{Gd}$ and ${ }^{152} \mathrm{Dy}$ experimental spin assignments from the links are inconsistent. This paper reports the search for links between SD and ND states in another $A \approx 150$ nucleus, namely ${ }^{151} \mathrm{~Tb}$.

Both ND [10,11] and SD [12-16] states in the ${ }^{151} \mathrm{~Tb}$ nucleus have been studied in considerable detail. Several studies on the decay-out from the yrast SD band in the ${ }^{151} \mathrm{~Tb}$ nucleus have been reported [17,18] and the first identification of a possible SD to ND linking transition (2818 keV) was found in the analysis of an experiment performed on 
EUROBALL IV $[19,20]$. However the statistics collected during $4.8 \mathrm{~d}$ was insufficient to fit the $2818 \mathrm{keV}$ transition into the decay scheme. Therefore a much longer experiment was performed in which many candidates for linking transitions were identified. Two of the strongest candidates, the $2818 \mathrm{keV}$ and the $3748 \mathrm{keV}$ gamma rays, have been shown to be single step decays from a level in the SD band to two ND levels with spins of $71 / 2^{-}$and $75 / 2^{-}$in ${ }^{151} \mathrm{~Tb}$.

\section{EXPERIMENTAL CONDITIONS}

The ${ }^{151} \mathrm{~Tb}$ nuclei were produced via the ${ }^{130} \mathrm{Te}\left({ }^{27} \mathrm{Al}, \mathrm{xn}\right)$ reaction at a beam energy of $155 \mathrm{MeV}$. A stack of two selfsupporting ${ }^{130} \mathrm{Te}$ foils $\sim 500 \mu \mathrm{g} \mathrm{cm}^{-2}$ thick were bombarded by an ${ }^{27} \mathrm{Al}$ beam produced at the Vivitron accelerator of the Institut de Recherches Subatomiques of Strasbourg, France. The $\gamma$-ray decays of the residual nuclei were measured using the Euroball IV array which consisted of 30 tapered Ge detectors (forward quadrant), 26 clover Ge detectors (around $90^{\circ}$ ) and 15 cluster Ge detectors (backward angles). Each Ge detector was surrounded by a BGO suppression shield. There was also an inner ball of BGO detectors (210 crystals), which was analysed by forming 164 groups of 'equivalent' detectors of approximately equal solid angle and efficiency, thus enabling a measurement of the multiplicity and the sum energy of $\gamma$-rays emitted in the nuclear reaction.

The data collection trigger for high-multiplicity events was that at least six unsuppressed (equivalent to four suppressed) Ge detectors and at least ten BGO ball elements fired. A total yield of $8.7 \times 10^{9}$ fourfold and higher-fold events was obtained with a mean fold of five. A nonspiked database according to the procedure described in Ref. [21] was used for the analysis of the large data set.

\section{RESULTS}

A triple-gated spectrum, shown in Fig. 1, was obtained using only clean ${ }^{151} \mathrm{~Tb}$ yrast SD band transitions and an advanced background subtraction process [22]. The strongest high energy candidate for a linking transition is at $3748 \mathrm{keV}$ which has an intensity of $\sim 0.9 \%$ relative to the yrast SD band intensity. At this high energy the $3748 \mathrm{keV}$ transition is expected to be a single-step link. No statistically significant transitions were present above $3748 \mathrm{keV}$. However, many other candidates were observed in the $1.9-3.7 \mathrm{MeV}$ range and the energies of the stronger lines are indicated on Fig. 1.

The next step is to identify the ND states to which the $3748 \mathrm{keV}$ transition decays. The relevant spectrum was obtained by adding a third single gate on the $3748 \mathrm{keV} \gamma$-ray to the multiple two gates on SD band transitions and part of the resulting spectrum is shown in Fig. 2(b). Figure 2(a) shows part of the spectrum arising from just two gates on the SD band transitions. In this spectrum the 3748 gate area contains 1300 counts in the $3748 \gamma$-ray itself but this is only $7 \%$ of the total counts in the gate. Therefore the background to be subtracted from the $2 \mathrm{SD}+3748$ spectrum is large and it is difficult to identify the appropriate background spectrum. The technique used above [22] is inappropriate as we are interested in the

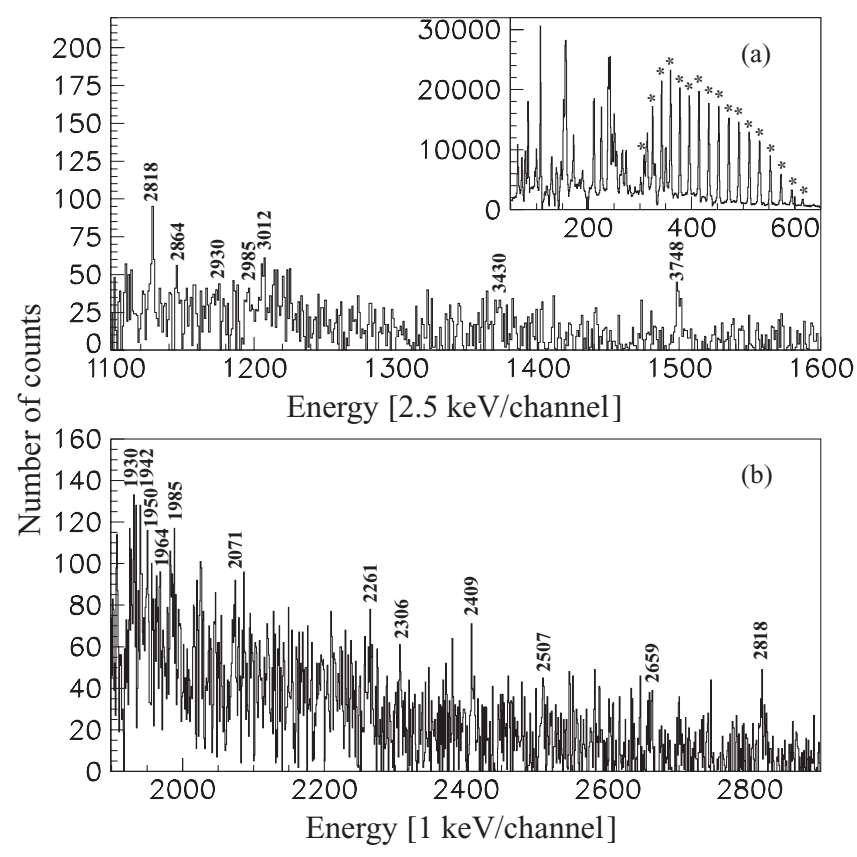

FIG. 1. (a) Parts of the $0-20 \mathrm{MeV}$ spectrum in coincidence with three gates on the ${ }^{151} \mathrm{~Tb} \mathrm{SD}$ transitions. In inset is displayed the lowenergy part of the 0-20 MeV spectrum showing the SD transitions (indicated by stars) and ND transitions fed by the yrast SD band. (b) Part of the $0-4 \mathrm{MeV}$ spectrum in coincidence with three gates on the ${ }^{151} \mathrm{~Tb} \mathrm{SD}$ transitions.

intensities of the ND transitions at high spin into which the 3748 transition decays. The intensities of these gamma-rays decrease rapidly with each addition of another SD gate as they are not fed in the majority of SD band decays. So the method of [22] would produce too much subtraction for these high spin ND transitions and result in negative peaks. It was concluded that it was preferable to base the analysis on the spectra without any background subtraction correction as it is the change in the individual high spin ND $\gamma$-ray intensities between the 2SD gated spectrum and the $2 \mathrm{SD}+3748$ gated spectrum that provides information on those transitions that are associated with the additional gate on the $3748 \mathrm{keV}$ transition. The intensities of the transitions between the ND states at the entry regime from the SD band were normalised to the low spin transitions at the bottom of the ND structure for the SD+SD spectrum, the $\mathrm{SD}+\mathrm{SD}+3748$ spectrum and the $\mathrm{SD}+\mathrm{SD}+\mathrm{SD}$ spectrum. The relative change in intensity when a third gate (either another SD gate or the 3748 gate) was added to the SD+SD spectrum provide the data for identifying the entry point into the ND states.

The $1096 \mathrm{keV}$ and $381 \mathrm{keV}$ ND transitions show a small increase as the relative intensity is 1.2(1) with the 3748 additional condition. The relative intensity is larger still at 1.4(2) for the higher spin $371 \mathrm{keV}$ and $1041 \mathrm{keV}$ transitions and then drops to 0.4(2) for the $934 \mathrm{keV}$ transition. The spectrum produced with the extra $\mathrm{SD}$ gate $(\mathrm{SD}+\mathrm{SD}+\mathrm{SD})$ shows a different behavior as the relative intensity commences at $0.95(15)$ for the $381 \mathrm{keV}$ transition, drops to just over 0.6 for the $1096 \mathrm{keV}$ and $371 \mathrm{keV}$ transitions and then to $0.5(1)$ for both the $1041 \mathrm{keV}$ and $934 \mathrm{keV}$ transitions. This reduction 


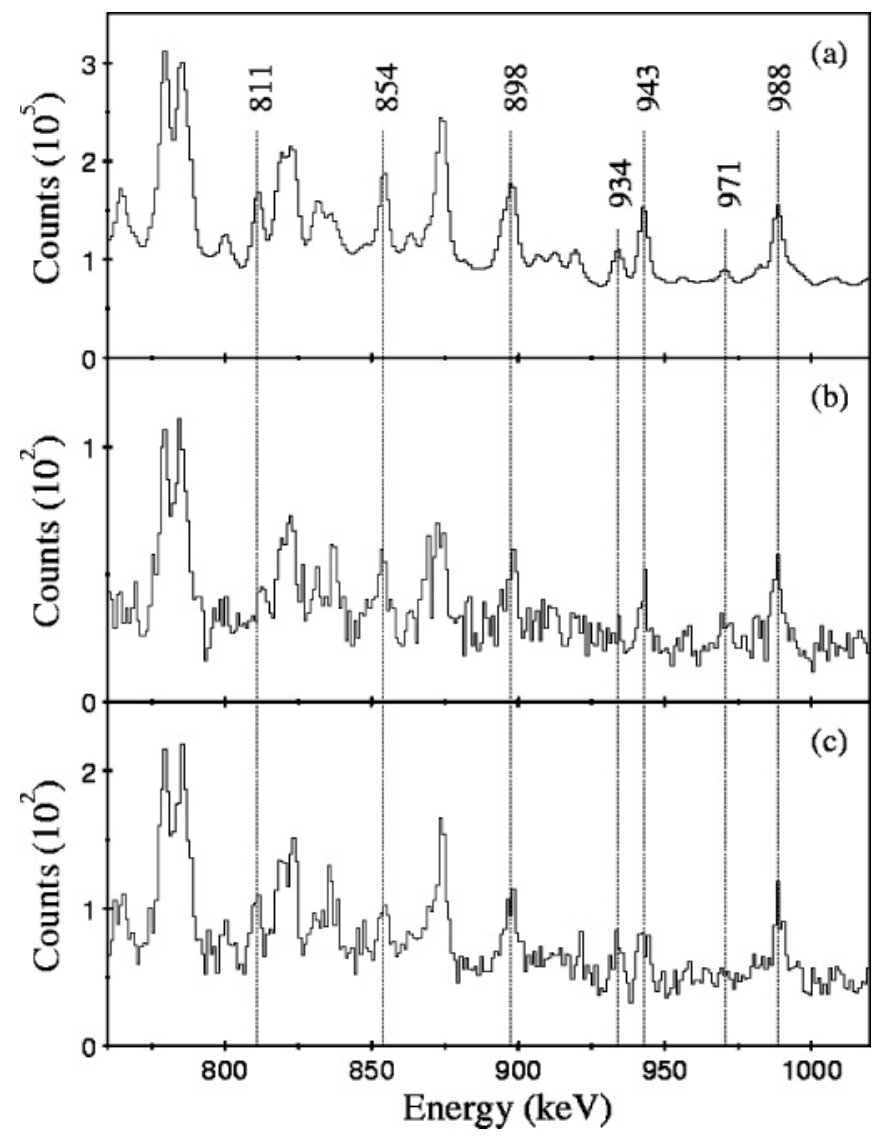

FIG. 2. (a) Spectrum obtained by setting pairwise gates on an SD transition. (b) Spectrum obtained from setting pairwise gates on an SD transition (above $854 \mathrm{keV}$ ) and an additional gate on the $3748 \mathrm{keV}$ transition. (c) Spectrum obtained from setting pairwise gates on an SD transition (above $854 \mathrm{keV}$ ) and an additional gate on the $2818 \mathrm{keV}$ transition.

in the intensities for the extra SD gate is because the majority of the decay paths from the SD band feed into the ND states at or below the $381 \mathrm{keV}$ transition. Indeed, in the spectrum generated from $4 \mathrm{SD}$ gates the relative intensities of the $371 \mathrm{keV}$ and $1041 \mathrm{keV}$ transitions are less than 0.1 and so this means that relative intensities of around 0.5 for the $1041 \mathrm{keV}$ and $934 \mathrm{keV} \gamma$-rays in the SD+SD+SD spectrum are entirely due to background coincidences. Thus the figure of 0.4(2) for the $934 \mathrm{keV}$ transition in the spectrum of SD+SD+3748 is consistent with this transition not being in the decay path of the $3748 \mathrm{keV}$ link. As the relative intensities of the $1096 \mathrm{keV}$, $371 \mathrm{keV}$, and $1041 \mathrm{keV}$ transitions are larger than unity it is concluded that these are in the decay path of the $3748 \mathrm{keV}$ link. Therefore the data clearly indicate that the $3748 \mathrm{keV}$ link decays into the ND states below the $934 \mathrm{keV}$ transition and into the $10690 \mathrm{keV}\left(71 / 2^{-}\right)$state. This assignment means that the energy of the SD state from which the $3748 \mathrm{keV} \gamma$-ray originates is $14440 \mathrm{keV}$.

Information was obtained on the multipolarity of the $3748 \mathrm{keV}$ linking transition from measurements of the angledependent intensity $I_{\gamma}(\theta)$ ratios using a method derived from directional correlations of decays from oriented nuclear states
(DCO ratio). An asymmetry ratio is defined as

$$
\begin{aligned}
R_{\mathrm{asym}} & =\frac{I_{\gamma}(\text { cluster }+ \text { tapered })}{I_{\gamma}(\text { clover })} \\
& \approx \frac{I_{\gamma}(\text { cluster })}{I_{\gamma}(\text { clover })} \approx \frac{I_{\gamma}(\text { backward })}{I_{\gamma}\left(90^{\circ}\right)},
\end{aligned}
$$

where $I_{\gamma}$ is the efficiency corrected intensity of the $\gamma$-ray detected with the cluster and clover detectors respectively. For comparison $R_{\text {asym }}$ values of known stretched $E 1$ (or $M 1$ ) transitions in the ND level scheme and $E 2$ transitions from the SD yrast band of the ${ }^{151} \mathrm{~Tb}$ nucleus have also been extracted; the mean values obtained are 0.58(2) and 1.11(2) for $E 1$ (or $M 1)$ and $E 2$ multipolarities, respectively. The value measured for the $3748 \mathrm{keV}$ transition is $0.52(0.11)$ which indicates a most probable dipole character for this transition. Without a linear polarization measurement, it is not possible to distinguish between magnetic or electric radiation. However, based on $E 1-M 1$ probabilities and also on similarities in the $A \approx 150$ mass region $[7,8]$, together with theoretical calculations that predict the yrast SD band has positive parity, we assume an $E 1$ character for this transition. Thus the $14440 \mathrm{keV}$ SD state from which the $3748 \mathrm{keV}$ transition originates has a spin of either $\left(73 / 2^{+}\right)$or $\left(69 / 2^{+}\right)$.

One approach to selecting the correct alternative between these two spin assignments is to identify other links between the SD band and the ND states. A link between the $14440 \mathrm{keV}$ SD state and the $9649 \mathrm{keV}\left(67 / 2^{-}\right.$) ND state (a $4791 \mathrm{keV}$ $\gamma$-ray) would establish the spin at the lower value of $\left(69 / 2^{+}\right)$. However no evidence was found for any $\gamma$-rays above $4 \mathrm{MeV}$. On the other hand, a link between the $14440 \mathrm{keV}$ SD state and the $11624 \mathrm{keV}\left(75 / 2^{-}\right) \mathrm{ND}$ state (a $2816 \mathrm{keV} \gamma$-ray) would establish the spin as $\left(73 / 2^{+}\right)$. As reported above a $2818(3) \mathrm{keV}$ $\gamma$-ray (intensity $\sim 0.7 \%$ relative to the yrast SD band intensity) was observed in the spectrum obtained from triple SD band gates as shown in Fig. 1.

In order to confirm that this $2818 \mathrm{keV} \gamma$-ray is the transition to the $11624 \mathrm{keV}$ ND state, the spectrum obtained by setting SD+SD+2818 gates (Fig. 2(c)) should show evidence of the $934 \mathrm{keV}$ transition from the $11624 \mathrm{keV}$ ND state. In the 2SD spectrum the 2818 gate contains 3200 counts in the 2818 $\gamma$-ray peak which is $4 \%$ of the total counts in the gate. In the $2 \mathrm{SD}+2818$ spectrum the $934 \mathrm{keV} \gamma$-ray is clearly present and its intensity relative to that of the same transition in the $\mathrm{SD}+\mathrm{SD}$ spectrum is 2.1(4). This figure is larger than the 1.3(2) observed for the subsequent $1041 \mathrm{keV}$ and $371 \mathrm{keV}$ transitions and 1.1(1) for the $1096 \mathrm{keV}$ and $371 \mathrm{keV}$ transitions. It is also very much larger than the relative intensity of $0.4(4)$ for the $934 \mathrm{keV} \gamma$-ray in the SD+SD+3748 spectrum. These data are strong evidence that the $2818 \mathrm{keV}$ link feeds the $11624 \mathrm{keV}$ ND state. Thus it is concluded that the $2818 \mathrm{keV}$ and $3748 \mathrm{keV} \gamma$-rays originate from the same SD level which has a spin of $\left(73 / 2^{+}\right)$and an energy of $14440 \mathrm{keV}$.

We now have to determine the SD state from which the $2818 \mathrm{keV}$ and $3748 \mathrm{keV}$ transitions decay. The spectra in Fig. 2(b) and 2(c) were produced in an analysis using SD band transition gates which did not include gates on the $811 \mathrm{keV}$ and $854 \mathrm{keV}$ SD $\gamma$-rays. Figure 2(b) (SD+SD+3748) shows the $\mathrm{SD}$ transitions down to the $854 \mathrm{keV}$ are clearly present with 

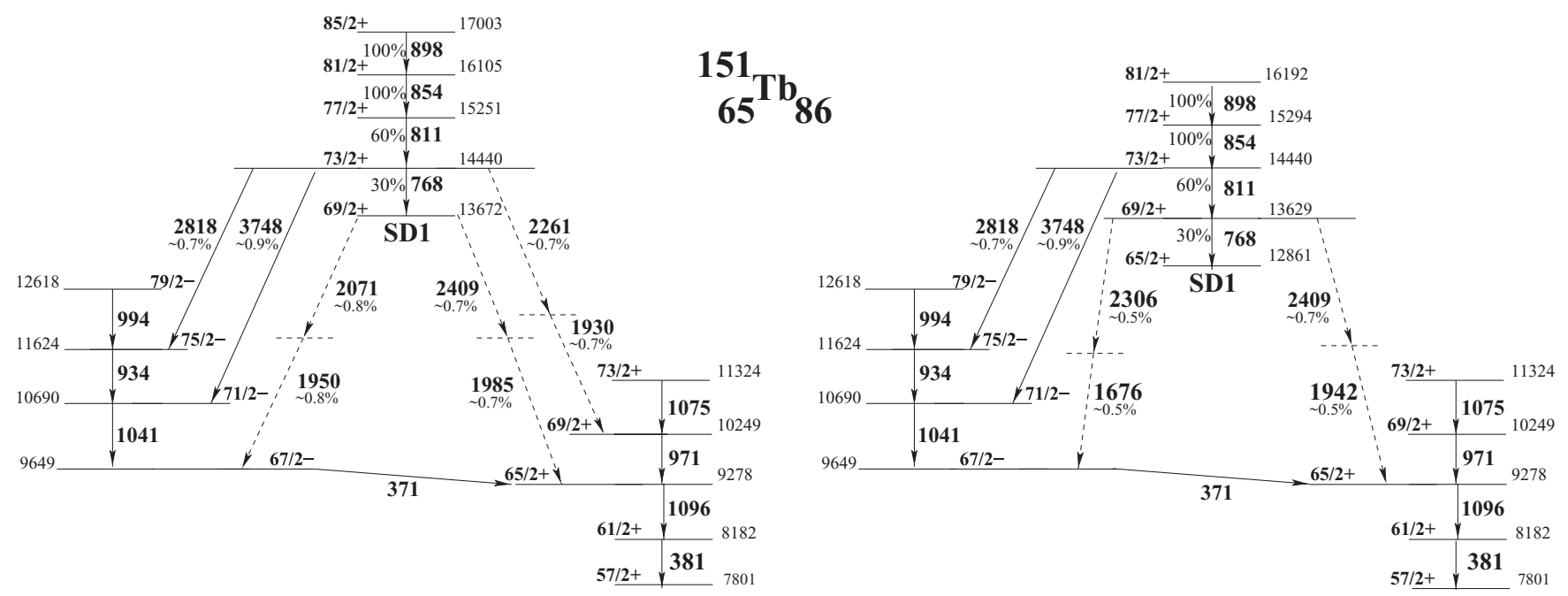

FIG. 3. Partial decay scheme of ${ }^{151} \mathrm{~Tb}$ showing the lowest part of the yrast SD band and ND states to which the SD band mainly decays for both of the decay scenarii.

the intensity of the $854 \mathrm{keV}$ transition 0.9(2) that of the higher energy SD transitions. There is a peak around $811 \mathrm{keV}$ and its intensity is only 0.4(3) relative to that of the high-energy SD transitions and this is consistent with the random background level as discussed above for the $934 \mathrm{keV}$ transition. Thus this spectrum indicates that the links decay out above the $811 \mathrm{keV}$ SD transition. Figure 2(c) (SD+SD+2818) shows a much stronger $811 \mathrm{keV} \gamma$-ray with an intensity relative to the higher energy SD transitions of 0.9(3) which is statistically consistent with it being in the decay path. Thus the 3748 gated spectrum apparently indicates that the link is before the $811 \mathrm{keV}$ SD transition whereas the 2818 gated spectrum indicates the link is after the $811 \mathrm{keV}$ transition. One difference between the backgrounds in the linking transition gates in the 2SD spectrum is that the higher background in the 2818 gate contains other $\gamma$-rays associated with the overall decay-out from the SD band whereas it is less likely that there are other single step $\gamma$-rays around $3748 \mathrm{keV}$. This effect could account for some of the intensity in the $811 \mathrm{keV}$ peak in the $2 \mathrm{SD}+2818$ spectrum. However the statistical uncertainties in both measurements are large and both data points are within 2 standard deviations of them being consistent with the opposite conclusion about the $811 \mathrm{keV} \gamma$-ray. Therefore it is concluded that an unambiguous assignment of the decay out point is not possible with the current data set and the two possibilities for the spin of the SD band head are $69 / 2^{+}$if the $811 \mathrm{keV} \gamma$-ray is in the linking path, and $65 / 2^{+}$if it is not in the path.

An attempt was made to see if any of the other $\gamma$-rays identified as possible links in the spectrum of Fig. 1 had the appropriate energies to create two-step decays from the SD band to the known ND states. From theoretical calculations and previous data the intensity of the second-step $\gamma$-ray is expected to be much weaker than the first-step due to the large number of possible final states. No combinations could be found with these conditions. However, there is a pair of equal intensity $\gamma$-rays $(1930 \mathrm{keV}+2261 \mathrm{keV})$ which have the correct sum energy to fit the energy difference between the $14440 \mathrm{keV}$ SD level $\left(73 / 2^{+}\right)$and the $10249 \mathrm{keV}$ ND level $\left(69 / 2^{+}\right)$.
Both transitions would be of $E 1$ character. As this possible branch decays from the same SD level as the $2818 \mathrm{keV}$ and $3748 \mathrm{keV}$ links it does not add any further information about the decay-out point in the SD band. That requires a decay branch from another SD level. If the SD band head has the higher spin of $69 / 2^{+}$there is a $2409 \mathrm{keV}$ and $1985 \mathrm{keV}$ combination that fits a link to the $9278 \mathrm{keV}\left(69 / 2^{+}\right)$ND level. However there is also a $2409 \mathrm{keV}$ and $1942 \mathrm{keV}$ combination that fits the related link, from the $13629 \mathrm{keV}\left(69 / 2^{+}\right) \mathrm{SD}$ level to the $9278 \mathrm{keV}\left(69 / 2^{+}\right) \mathrm{ND}$ level, if the SD band head has the lower spin. These two-step links, together with others to the $9649 \mathrm{keV}\left(67 / 2^{-}\right)$ND level, are shown in Fig. 3. As none of these two-step paths could be confirmed by coincidence relationships it is concluded that these two-step links do not assist in fixing the spins of the SD band.

\section{COMPARISON WITH ASSIGNMENTS IN ${ }^{152}$ Dy AND ${ }^{149}$ Gd}

The observation of $\gamma$-rays linking the yrast SD band in ${ }^{151} \mathrm{~Tb}\left(\pi[651] 3 / 2(r=+i)^{-1} \nu 7^{2}\right)$ with the ND states is the third case in the $A \approx 150$ region. It is important to establish whether either of the resulting proposed assignments in ${ }^{151} \mathrm{~Tb}$ are consistent with those previously proposed for the yrast SD bands in ${ }^{149} \mathrm{Gd}\left((\pi[651] 3 / 2)^{-2} \nu[770] 1 / 2(r=-i)^{-1}\right)$ and ${ }^{152}$ Dy $\left(\pi 6^{4} \nu 7^{2}\right)$ and whether they are in agreement with theoretical predictions.

Two major theoretical studies of the spins of SD bands in $\mathrm{Gd}, \mathrm{Tb}$, and Dy nuclei are the cranked relativistic mean field calculations (CRMF) by Afanasjev et al. [9] and the cranking calculations based on a Woods-Saxon potential (CWS) by El Aouad et al. [23] who also carried out Hartree-Fock calculations with Skyrme SkM* interactions. These studies together with earlier cranked Nilsson model calculations of Ragnarsson et al. [24] are in complete agreement on the high-N proton and neutron orbital configurations of the yrast bands and they provide an excellent comparison of the relative spin 
assignments between the yrast SD bands based on their relative effective spin alignments as shown in Table 2 of Afanasjev et al. The initial disagreement with Ragnarsson et al. on the spins in the Dy nuclei was not due to the theoretical calculations but the selection of the best set of spins for the experimental data. These spin sets differ by $2 \hbar$ and the difficulty was related to the influence of pairing which is large at low frequencies. It is now agreed that the lower spin choice of Afanasjev et al. [9] is correct.

The calculations also give predictions for the specific spin assignments and these are easier to discuss by using the example of the yrast SD band in ${ }^{152}$ Dy. Both Afanasjev et al. and El Aouad et al. do not include pairing effects and, in that scenario, they predict that the lowest energy state observed in the yrast SD band in ${ }^{152}$ Dy should be $26 \hbar$. However when El Aouad et al. include pairing in the CWS approach the spin is lowered to $24 \hbar$. It is this lower spin assignment which agrees with the experimental assignment of Lauritsen et al. [8]. It is also the spin assignment taken as the basis by Afanasjev et al. (Table 2) for the set of consistent spin assignments for the various high-N proton and neutron configurations in the SD bands of $\mathrm{Gd}, \mathrm{Tb}$, and Dy nuclei.

Based on the $24 \hbar$ spin for the band head in ${ }^{152}$ Dy it follows that the predicted spin assignment for the band head of the yrast SD band in ${ }^{151} \mathrm{~Tb}$ (the state fed by the $768 \mathrm{keV}$ gamma ray) is $65 / 2^{+}$. This prediction corresponds to the case in which the linking $\gamma$-rays arise from above the $811 \mathrm{keV} \mathrm{SD}$ transition and it is the lower of the two possible spin assignments (see discussion in the previous section). It is important to consider whether the experimental spin assignment in the yrast SD band in ${ }^{152}$ Dy could be $2 \hbar$ higher as this would equate to the other spin option from the data on ${ }^{151} \mathrm{~Tb}$. The ${ }^{152} \mathrm{Dy}$ experiment of Lauritsen et al. [8] was not only much longer than the current experiment thus producing better statistics, but it was able to use an efficient isomer trigger which greatly reduced the background from other reaction channels. These advantages produced spectra which clearly demonstrate that the $4011 \mathrm{keV}$ link in ${ }^{152}$ Dy can be unambiguously placed in the ${ }^{152}$ Dy level scheme. This link by itself does allow a second spin option which was rejected by the observation of much weaker candidates for other links. However this second spin option is $2 \hbar$ lower and not $2 \hbar$ higher as required to be consistent with the ${ }^{151} \mathrm{~Tb}$ links arising below the $811 \mathrm{keV}$ SD transition. Therefore we conclude that the overall evidence points to the SD band head in ${ }^{151} \mathrm{~Tb}$ being $65 / 2^{+}$. The ${ }^{151} \mathrm{~Tb}$ data also confirms the conclusion of Lauritsen et al. [8] from spectra of other links with poorer statistics that the $4011 \mathrm{keV}$ link in ${ }^{152}$ Dy cannot be associated with the second spin option which is $2 \hbar$ lower.

Afanasjev et al. predicted that the spin assignment for the yrast SD band head in ${ }^{149} \mathrm{Gd}$ is $51 / 2^{-}$. However Finck et al. [7] proposed that it is $2 \hbar$ lower which is inconsistent with the predicted value. In ${ }^{149} \mathrm{Gd}$ the link is not a single step but involves two $\gamma$-rays and assumes a spin change of $3 \hbar$ with the first $2188 \mathrm{keV} \gamma$-ray of $E 1$ character and the second $2585 \mathrm{keV}$ $\gamma$-ray of $E 2$ character. As $M 2, E 3$ and other higher multipole transitions are extremely unlikely it is concluded that there can be no further increase in the spin change of $3 \hbar$ assumed for the two-step link. The stronger first-step $\gamma$-ray has a much larger intensity and its link with the SD band was clearly identified whereas the link into the ND states of the weaker second-step was not established but inferred from the overall linkage of the first-step $2188 \mathrm{keV} \gamma$-ray into the ND states. This spectrum showed only weak feeding into the 49/2 state which has a mean lifetime of $4 \mathrm{~ns}$ and decays with a $186 \mathrm{keV}$ E2 transition. On this evidence Finck et al. [7] proposed that the $2585 \mathrm{keV}$ second step fed into the ND states below the $186 \mathrm{keV}$ transition. However a second possibility is consistent with the experimental data. The weak $186 \mathrm{keV}$ line observed in Ref. [7] could have been due to the very short flight time for the recoiling nuclei $(<0.5 \mathrm{~ns})$ before they were blocked by the Ge-detector collimator which meant that only about $\sim 5 \%$ of the emitted $186 \mathrm{keV}$ transitions were detected. In this scenario the $2585 \mathrm{keV} \gamma$-ray is the weak feeding of the strong $2188 \mathrm{keV}$ first-step link into the 49/2 isomer state and thus the spin assignments of the SD band head in ${ }^{149} \mathrm{Gd}$ would be $2 \hbar$ higher making them consistent with the predicted values.

The relative energies of the ND and SD structures as a function of spin is a critical input to calculations which attempt to model the feeding of the SD bands at high spins. In particular the spin at which the energies of the yrast ND and yrast SD are equal has been used as a useful parameter. In the case of ${ }^{152}$ Dy this was considered to be around a spin of $54 \hbar[25,26]$. The relative energies of the yrast superdeformed and low deformation bands plus the yrast single-particle states are shown in Fig. 4 for ${ }^{151} \mathrm{~Tb}$ and ${ }^{152} \mathrm{Dy}$. The situation is unique in ${ }^{152}$ Dy as three structures have been observed and the low deformation band, which is proposed to have a triaxial shape [27], does cross the SD band. The crossing spin is low at $42 \hbar$. Experimentally the single-particle states in ${ }^{152}$ Dy have only been observed up to $36 \hbar$ but theoretical calculations [28] predict that their relative energies will increase by over $1 \mathrm{MeV}$ around $40 \hbar$ due to excitations across the 82 shell gap. An increase of only $0.5 \mathrm{MeV}$ would bring the crossing point with the SD band to below $50 \hbar$ and at $1 \mathrm{MeV}$ it would be at $46 \hbar$. This latter value would be consistent with the observation that the low deformation band is strongly fed between $40-46 \hbar$ as its excitation energy relative to the single-particle states would then be around $1 \mathrm{MeV}$. Thus it is concluded that at high spins
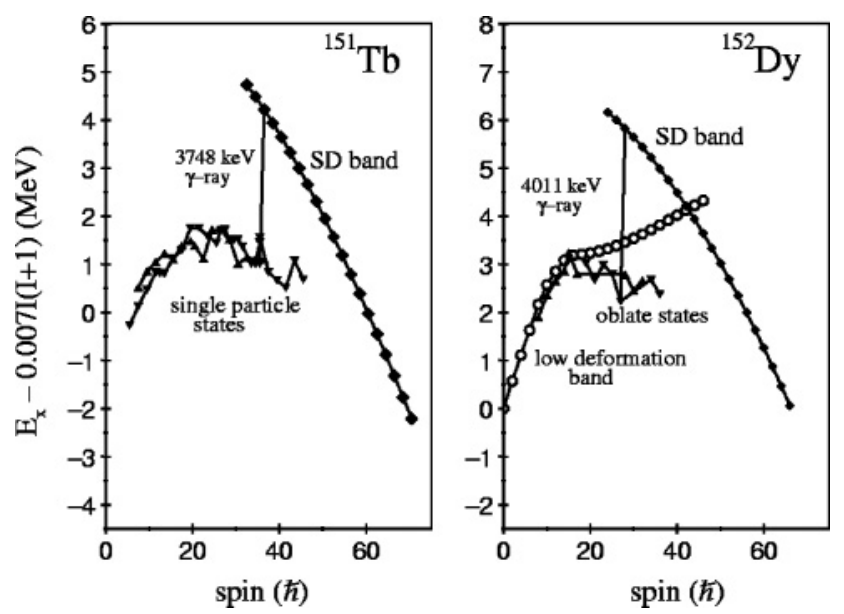

FIG. 4. Relative energies of the yrast superdeformed (squares) and low deformation (circles) bands in ${ }^{151} \mathrm{~Tb}$ and ${ }^{152} \mathrm{Dy}$. The yrast single-particle states (triangles) are also indicated. 
the yrast $\mathrm{SD}$ band in ${ }^{152} \mathrm{Dy}$ is much lower in energy relative to the ND states than predicted.

In ${ }^{151} \mathrm{~Tb}$ the single-particle states are known up to a higher spin of over $45 \hbar$. Assuming an increase of $1 \mathrm{MeV}$ due to excitations across the shell gap, the single-particle states would cross the SD band at around $52 \hbar$ which is $6 \hbar$ higher than in ${ }^{152} \mathrm{Dy}$. This spin increase is replicated in theoretical calculations [26] as the crossing point goes from $54 \hbar$ in ${ }^{152} \mathrm{Dy}$ to $58 \hbar$ in ${ }^{151} \mathrm{~Tb}$ and it underlines the role of large shell gaps in both protons and neutrons in ${ }^{152} \mathrm{Dy}$.

In summary, two transitions have been identified that link a state in the yrast SD band to two specific ND states in ${ }^{151} \mathrm{~Tb}$. Current experimental evidence cannot unambiguously determine between the two possible alternatives for the SD state. However based on theoretical relative spin predictions of yrast SD bands in the $A \approx 150$ region it was concluded that the alternative that resulted in a lower spin for the SD band head in ${ }^{151} \mathrm{~Tb}$ was consistent with assignments in other nuclei. An order of magnitude increase in statistics will be needed to confirm this assignment directly from an experiment on ${ }^{151} \mathrm{~Tb}$ and this should be feasible using the next generation of gamma-ray spectrometers currently under development.

\section{ACKNOWLEDGMENTS}

The Euroball project was a collaboration between Denmark, France, Germany, Italy, Sweden, and the United Kingdom. This work was partially supported by the EU (Contract No. EUROVIV: HPRI-CT-1999-00078).
[1] P. J. Twin et al., Phys. Rev. Lett. 57, 811 (1986).

[2] X. Han and C. Wu, At. Data Nucl. Data Tables 73, 43 (1999).

[3] T. L. Khoo et al., Phys. Rev. Lett. 76, 1583 (1996).

[4] D. P. McNabb et al., Phys. Rev. C 56, 2474 (1997).

[5] S. Perries et al., Z. Phys. A 356, 1 (1996).

[6] A. Lopez-Martens et al., Phys. Lett. B380, 18 (1996).

[7] C. Finck et al., Phys. Lett. B467, 15 (1999).

[8] T. Lauritsen et al., Phys. Rev. Lett. 88, 042501 (2002).

[9] A. V. Afanasjev et al., Nucl. Phys. A634, 395 (1998).

[10] D. Curien, Ph.D. thesis no. 441, Université Louis Pasteur Strasbourg (1988).

[11] C. M. Petrache et al., Nucl. Phys. A579, 285 (1994).

[12] T. Byrski et al., Phys. Rev. Lett. 64, 1650 (1990).

[13] B. Kharraja et al., Phys. Lett. B341, 268 (1995).

[14] J. Robin et al., Phys. Rev. C 77, 014308 (2008).

[15] C. Finck et al., Eur. Phys. J. A 2, 123 (1998).
[16] G. Benzoni et al., Phys. Rev. C 75, 047301 (2007).

[17] D. Curien et al., Phys. Rev. Lett. 71, 2559 (1993).

[18] C. M. Petrache et al., Phys. Scr. T56, 299 (1995).

[19] A. Odahara et al., First joint meeting of the nuclear physics division of APS and JPS, Hawaii, USA, Oct. 2001.

[20] J. Robin et al., Symposium on Nuclear Structure Physics with Euroball: achievements 1997-2002, Paris-Sud university, Orsay Campus, March 2002.

[21] O. Stezowski, Ch. Finck, and D. Prévost, Nucl. Instrum. Methods. A 424, 552 (1999).

[22] J. Robin, Nucl. Instrum. Methods A 555, 282 (2005).

[23] N. El. Aouad et al., Nucl. Phys. A676, 155 (2000).

[24] I. Ragnarsson et al., Nucl. Phys. A557, 167c (1993).

[25] K. Schiffer et al., Z. Phys. A 332, 17 (1989).

[26] T. R. Werner and J. Dudek, Phys. Rev. C 44, R948 (1991).

[27] D. E. Appelbe et al., Phys. Rev. C 66, 044305 (2002).

[28] I. Ragnarsson et al., Phys. Lett. B180, 191 (1986). 